\title{
The Lawson homology for Fulton-MacPherson configuration spaces
}

WENCHUAN HU

LI LI

In this paper, we compute the Lawson homology groups and Deligne-Beilinson cohomology groups for the Fulton-MacPherson configuration spaces.

14F43, 55R80

\section{Introduction}

The purpose of this paper is to give formulas for the Lawson homology groups and Deligne-Beilinson cohomology groups of the Fulton-MacPherson configuration spaces $X[n]$ for $n \in \mathbb{N}$. The Lawson homology groups of $X[n]$ can be decomposed as a direct sum of Lawson homology groups of certain Cartesian products of $X$ with shifts of bidegrees. A similar decomposition holds for Deligne-Beilinson cohomology groups.

All varieties in the paper are defined over $\mathbb{C}$. Let $X$ be a $d$-dimensional projective variety and let $\mathcal{Z}_{p}(X)$ be the space of algebraic $p$-cycles on $X$. The Chow group $\mathrm{Ch}_{p}(X)$ of $p$-cycles is defined to be $\mathcal{Z}_{p}(X)$ modulo the rational equivalence (see Fulton [8, Section 1.3]). The Lawson homology $L_{p} H_{k}(X)$ of $p$-cycles is defined as

$$
L_{p} H_{k}(X):=\pi_{k-2 p}\left(\mathcal{Z}_{p}(X)\right) \text { for } k \geq 2 p \geq 0,
$$

where $\mathcal{Z}_{p}(X)$ is provided with a natural compactly generated topology (see Friedlander [6] and Lawson [13]). Set $L_{p} H_{k}(X)=L_{0} H_{k}(X)$ for $p<0$. (See Lawson [14] for the general background on Lawson homology.)

Let $F(X, n) \subset X^{n}$ be the complement of the diagonals, ie,

$$
F(X, n):=\left\{\left(x_{1}, \ldots, x_{n}\right) \in X^{n}: x_{i} \neq x_{j}, \forall i \neq j\right\} .
$$

For each subset $I \subseteq[n]:=\{1, \ldots, n\}$ with at least two elements, denote by $\mathrm{Bl}_{\Delta}\left(X^{I}\right)$ the blow-up of the Cartesian product $X^{I}$ along its small diagonal (for example when $I=\{1,4,5\}, X^{I}=\left\{\left(x_{1}, x_{4}, x_{5}\right)\right\} \cong X^{3}$ and its small diagonal $\Delta$ consists of points satisfying $\left.x_{1}=x_{4}=x_{5}\right)$. The Fulton-MacPherson configuration space is defined as follows. 
Definition-Theorem 1.1 (Fulton-MacPherson [9, Theorems 1 and 3]) Let $X$ be a smooth projective variety over $\mathbb{C}$. The closure of the natural locally closed embedding

$$
F(X, n) \hookrightarrow X^{n} \times \prod_{|I| \geq 2} \mathrm{Bl}_{\Delta}\left(X^{I}\right)
$$

is nonsingular, and the boundary is a simple normal crossing divisor. The closure is called the Fulton-MacPherson configuration space and is denoted by $X[n]$.

Let us introduce some combinatorial definitions first.

Definition 1.1 Fix a positive integer $n$. Denote $[n]:=\{1, \ldots, n\}$.

Two subsets $I, J \subseteq[n]:=\{1,2, \ldots, n\}$ are called overlapped if $I \cap J$ is a nonempty proper subset of $I$ and of $J$.

A nest $\mathcal{S}$ of $[n]$ is a set of subsets of $[n]$ such that each subset $I \in \mathcal{S}$ contains at least two elements and any two subsets $I, J \in \mathcal{S}$ are not overlapped.

Next we explain some definitions in graph theory. All trees in the paper will be rooted trees.

Definition 1.2 A (rooted) tree is a finite graph in which any two nodes are connected by exactly one path, together with a root node.

The tree-order is the partial ordering on the nodes of a tree with $u<v$ if and only if $u \neq v$ and the unique path from the root to $\mathrm{v}$ passes through $\mathrm{u}$. (So the root is the minimal node in a tree.)

A forest is a disjoint union of trees; it has a natural partial order induced from the tree-order. (A tree is a forest by definition.)

A leaf is a maximal node in a forest.

In a forest, a node $v$ is called a child of a node $u$ if $u<v$ and there is no node $w$ satisfying $u<w<v$.

We will explain in Section 2.2 that there is a 1-1 correspondence between the set of nests of $[n]$ and the set of forests with $n$ leaves, such that each element of the nest labels a node of the forest.

Definition 1.3 Let $\mathcal{S}$ be a nest of $[n]$.

Define $c(\mathcal{S})$ to be the number of connected components of the forest corresponding to $\mathcal{S}$. 
Define $c_{I}(\mathcal{S})$ (or $c_{I}$ if no ambiguity arises) to be the number of children of the node with label $I$.

For a nonempty nest $\mathcal{S}$, define the set $M_{\mathcal{S}}$ of lattice points in the integer lattice $\mathbb{Z}^{\mathcal{S}}$ as

$$
M_{\mathcal{S}}:=\left\{\underline{\mu}=\left(\mu_{I}\right)_{I \in \mathcal{S}}: 1 \leq \mu_{I} \leq\left(c_{I}-1\right) \operatorname{dim} X-1, \mu_{I} \in \mathbb{Z}\right\} .
$$

Define the norm $\|\mu\|:=\sum_{I \in \mathcal{S}} \mu_{I}$ for $\mu \in M_{\mathcal{S}}$. For $\mathcal{S}=\varnothing$, we assume that there is only one lattice point $\underline{0}$ in $M_{\mathcal{S}}$ whose norm is 0 .

The first main theorem asserts that the Lawson homology group of $X[n]$ can be decomposed as a direct sum of the Lawson homology groups of the Cartesian products of $X$ with shifts of bidegrees.

Theorem 1.2 Let $X$ be a smooth projective variety defined over $\mathbb{C}$. Then for each pair of integers $p, k$ such that $k \geq 2 p \geq 0$, there is an isomorphism of Lawson homology groups

$$
L_{p} H_{k}(X[n]) \cong \bigoplus_{\mathcal{S}} \bigoplus_{\underline{\mu} \in M_{\mathcal{S}}} L_{p-\|\underline{\mu}\|} H_{k-2\|\underline{\mu}\|}\left(X^{c(\mathcal{S})}\right),
$$

where the direct sum $\bigoplus_{\mathcal{S}}$ run over all the nests $\mathcal{S}$ of $[n]$.

As a consequence of the above theorem, we obtain the following more explicit formula for $L_{p} H_{k}(X[n])$. We explain some notation first: generalizing $\mathrm{R}$ Stanley's notation $\left[x^{i}\right] F(x)$ in [20] (which gives the coefficient of $x^{i}$ in the power series $F(x)$ ) to two variables $x$ and $t$, we define $\left[x^{i} t^{n} / n !\right] F(x, t)=a_{i n}$ for a power series

$$
F(x, t):=\sum_{j, k} a_{j k} \frac{x^{j} t^{k}}{k !} .
$$

Theorem 1.3 Let $X$ be a smooth $d$-dimensional projective variety defined over $\mathbb{C}$. Then for each pair of integers $p, k$ such that $k \geq 2 p \geq 0$, there is an isomorphism of Lawson homology groups

$$
L_{p} H_{k}(X[n]) \cong \bigoplus_{\substack{1 \leq m \leq n \\ 0 \leq i \leq p}} L_{p-i} H_{k-2 i}\left(X^{m}\right)^{\oplus\left[x^{i} t^{n} / n !\right] N^{m} / m !}
$$

where $N:=N(x, t)=\sum_{i \geq 1} h_{i}(x)\left(t^{i} / i !\right)$ is the exponential generating function of polynomials $h_{i}(x)$ determined by the identity

$$
(1-x) x^{d} t+\left(1-x^{d+1}\right)=\exp \left(x^{d} N\right)-x^{d+1} \exp (N) .
$$

We also obtain a formula similar to Theorem 1.2 for Deligne-Beilinson cohomology. 
Theorem 1.4 Let $X$ be a smooth projective variety defined over $\mathbb{C}$. Then for each pair of integers $p, k$, there is an isomorphism of Deligne-Beilinson cohomology groups

$$
H_{\mathcal{D}}^{k}(X[n], \mathbb{Z}(p)) \cong \bigoplus_{\mathcal{S}} \bigoplus_{\underline{\mu} \in M_{\mathcal{S}}} H_{\mathcal{D}}^{k-2\|\underline{\mu}\|}\left(X^{c(\mathcal{S})}, \mathbb{Z}(p-\|\underline{\mu}\|)\right) .
$$

Similarly, as a consequence of the above theorem, we obtain the following more explicit formula for $H_{\mathcal{D}}^{k}(X[n], \mathbb{Z}(p))$.

Theorem 1.5 Let $X$ be a smooth $d$-dimensional projective variety defined over $\mathbb{C}$. Then for each pair of integers $p, k$, there is an isomorphism of Deligne-Beilinson cohomology groups

$$
H_{\mathcal{D}}^{k}(X[n], \mathbb{Z}(p)) \cong \bigoplus_{\substack{1 \leq m \leq n \\ 0 \leq i \leq p}} H_{\mathcal{D}}^{k-2 i}\left(X^{m}, \mathbb{Z}(p-i)\right)^{\oplus\left[x^{i} t^{n} / n !\right] N^{m} / m !}
$$

where $N$ is the same as in Theorem 1.3.

The key tools used to prove the main results are the blow-up formula for Lawson homology proved by the first author [10] and the method for computing the Chow groups of the Fulton-MacPherson configuration space of the second author [15]. The structure of the paper is as follows: In Section 2, we briefly review background material about Lawson homology, as well as the construction on Fulton-MacPherson spaces and we compute the Lawson homology groups of the Fulton-MacPherson configuration space thus constructed. In Section 3, we briefly review background material about Deligne-Beilinson cohomology and we compute the Deligne-Beilinson cohomology for the Fulton-MacPherson configuration space. In Section 4, we compare Lawson homology with integral singular homology using simple examples. In Section 5, we write down the formula in Theorem 1.3 explicitly for $n=2$ and $n=3$.

\section{Lawson homology groups of Fulton-MacPherson spaces}

In this section, we prove Theorem 1.2 and Theorem 1.3. According to the construction, the Fulton-MacPherson configuration space $X[n]$ is obtained by successively blowing up $X^{n}$ along (the strict transforms of) its diagonals in a suitable order, where each blow-up is along a nonsingular subvariety. Therefore, we can calculate the Lawson homology groups of $X[n]$ by successively applying the blow-up formula for Lawson homology (see Theorem 2.1). 


\subsection{Lawson homology}

Recall that for a morphism $f: W \rightarrow V$ between projective varieties, there exist induced homomorphisms $f_{*}: L_{p} H_{k}(W) \rightarrow L_{p} H_{k}(V)$ for all $k \geq 2 p \geq 0$. Furthermore, it has been shown that if $W$ and $V$ are smooth and projective, there are Gysin "wrong way" homomorphisms $f^{*}: L_{p} H_{k}(V) \rightarrow L_{p-c} H_{k-2 c}(W)$, where $c=\operatorname{dim}(V)-\operatorname{dim}(W)$ (see Peters [19]).

Let $X$ be a smooth projective variety and $Y$ be a smooth subvariety of $X$ of codimension $r$ with the natural embedding $i_{0}: Y \hookrightarrow X$. Let $\sigma: \tilde{X}_{Y} \rightarrow X$ be the blow-up of $X$ along $Y$, let $D$ be the exceptional divisor and $\pi: D \rightarrow Y$ and $i: D \hookrightarrow \tilde{X}_{Y}$ be the natural morphisms. Set $U:=X-Y \cong \tilde{X}_{Y}-D$. Denote by $j_{0}$ the inclusion $U \hookrightarrow X$ and $j$ the inclusion $U \hookrightarrow \tilde{X}_{Y}$. That is, we have a diagram:

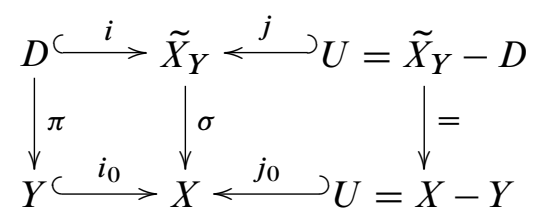

It is well known that $\pi: D \rightarrow Y$ gives a projective bundle of rank $r-1$ by identifying $D$ with $\mathbb{P}\left(N_{Y / X}\right)$. (Note: We adopted the "old fashioned" geometric notation for the projective bundle $\mathbb{P}(E)$ associated with a vector bundle $E$ used in Fulton's book [8, B.5.5], instead of Grothendieck's notation in EGA.)

Moreover, we have (see Voison [23, page 271])

$$
\left.\mathcal{O}_{\tilde{X}_{Y}}(D)\right|_{D}=\mathcal{O}_{\mathbb{P}\left(N_{Y / X}\right)}(-1) \text {. }
$$

Denote by $h$ the class of $\mathcal{O}_{\mathbb{P}\left(N_{Y / X}\right)}(-1)$ in $\operatorname{Pic}(D)$. We have $h=\left.[-D]\right|_{D}$ and $-h=i^{*} i_{*}: L_{q} H_{m}(D) \rightarrow L_{q-1} H_{m-2}(D)$ for $0 \leq 2 q \leq m$ [7, Theorem 2.4; 19, Lemma 11]. The last equality can be equivalently regarded as a Lefschetz operator

$$
-h=i^{*} i_{*}: L_{q} H_{m}(D) \rightarrow L_{q-1} H_{m-2}(D), \quad 0 \leq 2 q \leq m .
$$

The following result is essential to the proof of Theorem 1.2.

Theorem 2.1 (Lawson homology for a blow-up [10, Theorem 1.2]) Let $X$ be a smooth projective variety and $Y \subset X$ be a smooth subvariety of codimension $r$. Let $\sigma: \tilde{X}_{Y} \rightarrow X$ be the blow-up of $X$ along $Y, \pi: D=\sigma^{-1}(Y) \rightarrow Y$ the natural map, and $i: D=\sigma^{-1}(Y) \rightarrow \tilde{X}_{Y}$ the exceptional divisor of the blow-up. Then for each $p, k$ 
with $k \geq 2 p \geq 0$, we have the isomorphism

$$
I_{p, k}:\left\{\bigoplus_{1 \leq j \leq r-1} L_{p-j} H_{k-2 j}(Y)\right\} \oplus L_{p} H_{k}(X) \stackrel{\cong}{\longrightarrow} L_{p} H_{k}\left(\tilde{X}_{Y}\right)
$$

given by

$$
I_{p, k}\left(u_{1}, \ldots, u_{r-1}, u\right)=\sum_{j=1}^{r-1} i_{*}\left(h^{j} \cdot \pi^{*} u_{j}\right)+\sigma^{*} u .
$$

Remark 2.2 The above theorem proved in [10] can be generalized without difficulty to $l$-adic Lawson homology (see Friedlander [6]) for nonsingular projective varieties $X$ over any algebraic closed field of characteristic $p$ where $(p, l)=1$.

\subsection{The Fulton-MacPherson configuration spaces}

The Fulton-MacPherson configuration spaces $X[n]$ were discovered around 1989 [9]. In their original paper, Fulton and MacPherson used it to construct a differential graded algebra which is a model for $F(X, n)$ in the sense of Sullivan. Axelrod and Singer constructed a similar compactification in the setting of smooth manifolds $[1$, Section 5.1]. The space $\mathbb{P}^{1}[n]$ is closely related the Deligne-Mumford compactification $\bar{M}_{0, n}$ of the moduli space of nonsingular rational curves with $n$ marked points (see Keel [12]).

The following notation is needed. Fix a positive integer $n$. There is a 1-1 correspondence between the set of nests of $[n]$ and the set of forests with $n$ leaves which sends a nest $\mathcal{S}$ to a forest $F$ as follows: the $n$ leaves in $F$ are labeled by $1,2, \ldots, n$. Each element $I$ of $\mathcal{S}$ gives a node of $F$, labeled by $I$. Two nodes with labels $I$ and $J$ are connected by an edge if $I \subsetneq J$ and there does not exist $K \in \mathcal{S}$ such that $I \subsetneq K \subsetneq J$. Denote by $c(\mathcal{S})$ the number of connected components of the forest corresponding to $\mathcal{S}$ and denote by $c_{I}(\mathcal{S})$, or $c_{I}$, the number of children of the node with label $I$. Below is an example.

Example 2.3 Let $n=7$ and $\mathcal{S}=\{1234,12,56\}$. See Figure 1 .

In this example, $c(\mathcal{S})=3, c_{12}=c_{56}=2$ and $c_{1234}=3$.

For a nonempty nest $\mathcal{S}$, define the set $M_{\mathcal{S}}$ as in equation (1).

Example 2.4 Let $n=7$ and $\mathcal{S}=\{1234,12,56\}$ be as in Example 2.3 and let $d=$ $\operatorname{dim} X$. Then

$M_{\mathcal{S}}=\left\{\left(\mu_{1234}, \mu_{12}, \mu_{56}\right) \in \mathbb{Z}^{3}: 1 \leq \mu_{1234} \leq 2 d-1,1 \leq \mu_{12} \leq d-1,1 \leq \mu_{56} \leq d-1\right\}$. 


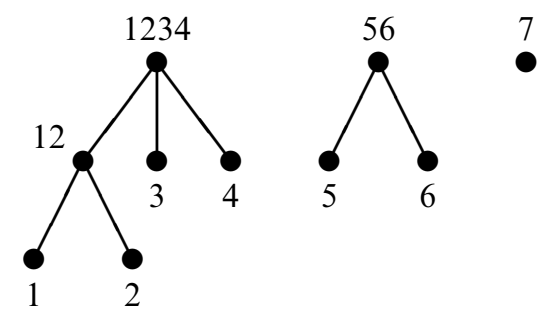

Figure 1: The forest corresponding to the nest $\mathcal{S}=\{1234,12,56\}$

Consider the following ordered set of diagonals

$$
\underbrace{\Delta_{12 \cdots n}}_{\left(\begin{array}{c}
n \\
n
\end{array}\right)}, \underbrace{\Delta_{12 \cdots(n-1)}, \Delta_{12 \cdots(n-2), n}, \ldots, \Delta_{23 \cdots n}}_{\left(\begin{array}{c}
n \\
n-1
\end{array}\right)}, \ldots, \underbrace{\Delta_{12}, \ldots, \Delta_{n-2, n}, \Delta_{n-1, n}}_{\left(\begin{array}{c}
n \\
2
\end{array}\right)}
$$

which induces an order on $\{I \subseteq[n]:|I| \geq 2\}$ :

$\{1,2, \ldots, n\}<\{1,2, \ldots, n-1\}<\{1,2, \ldots, n-2, n\}<\cdots<\{n-2, n\}<\{n-1, n\}$.

The following lemma is needed for the proof of Theorem 1.2. This lemma is implicit in De Concini and Procesi [3], MacPherson and Procesi [18, Section 5.1], and has been pointed out, but not as explicitly as below, in Thurston [21, Proposition 3.5 and 3.6] in the situation of real manifolds.

Lemma 2.5 $X[n]$ can be constructed by successively blowing up (the strict transforms of) the $2^{n}-n-1$ diagonals of $X^{n}$ in the order (2).

Proof A proof is given in [16, Proposition 2.13] by the second author and we will not reproduce it here. The idea is to prove inductively for a more general situation - the wonderful compactification of an arrangement - using the notion due to De Concini and Procesi of building sets of arrangements. The interested reader is referred to [16, Theorem 1.3] for a conclusion on orders of blow-ups in the construction of the wonderful compactification spaces, which in particular applies to Fulton-MacPherson spaces.

Proof of Theorem 1.2 Since the Fulton-MacPherson configuration space $X[n]$ can be constructed by a sequence of blow-ups, we can obtain its Lawson homology group by successively applying the formula of one blow-up (Theorem 2.1).

The notion of nest appears naturally when we decompose the Lawson homology groups of $X[n]$ in terms of Lawson homology groups of Cartesian products of $X$. Take $X^{n}$ as the ambient variety. For $I \subseteq[n]$ with at least two elements, it can be shown (as a 
special case of [15, Proposition 2.7]) that the strict transform $\widetilde{\Delta}_{I}$ (which will be blown up along) is obtained by successively blowing up $\Delta_{I}$ along the centers $\Delta_{J} \cap \Delta_{I}$ for ( $J \supsetneq I)$ or $(J<I$ and $J \cap I=\varnothing)$, so $\{I, J\}$ is a nest; take $\Delta_{I}$ as the ambient variety instead of $X^{n}$. The strict transform of $\Delta_{I} \cap \Delta_{J}$ (which will be blown up along which we blow up is obtained ) is obtained by successively blowing up $\Delta_{I} \cap \Delta_{J}$ along those $\Delta_{I} \cap \Delta_{J} \cap \Delta_{K}$ such that $K$ satisfies

- $\quad I \cap J \cap K=\varnothing$ and $K<J$,

- $K \supsetneq I$ and $K \cap J=\varnothing$ or

- $K \supsetneq J$ and $K \cap I=\varnothing$,

so the set $\{I, J, K\}$ is a nest. In general, consider a nest $\mathcal{S}=\left\{I_{1}, I_{2}, \ldots, I_{\ell}\right\}$ which is arranged in the order compatible with (2), that is, the elements in $\mathcal{S}$ is ordered as the order of $\Delta_{I_{j}}, 1 \leq j \leq \ell$ in (2). Then $\mathcal{S}$ determines a chain of polydiagonals (ie intersections of diagonals) of $X^{n}$ :

$$
\bigcap_{j=1}^{\ell} \Delta_{I_{j}} \subset \bigcap_{j=2}^{\ell} \Delta_{I_{j}} \subset \bigcap_{j=3}^{\ell} \Delta_{I_{j}} \subset \cdots \subset \bigcap_{j=\ell-1}^{\ell} \Delta_{I_{j}} \subset \Delta_{I_{\ell}} \subset X^{n} .
$$

The codimension of the $i$-th polydiagonal in the $(i+1)$-th polydiagonal equals $\left(c_{I_{i}}-1\right) \operatorname{dim} X$. Blowing up along the strict transform of $\Delta_{I_{i}}$ makes $\left(c_{I_{i}}-1\right) \operatorname{dim} X-1$ copies of the Lawson homology groups (with shifted bidegrees) of the strict transform of the $i$-th polydiagonal $\bigcap_{j=i}^{\ell} \Delta_{I_{j}}$ contributing to the Lawson homology group of the strict transform of the $(i+1)$-th polydiagonal $\bigcap_{j=i+1}^{\ell} \Delta_{I_{j}}$. By successively applying the formula of a blow-up (Theorem 2.1), one sees that for each lattice point $\left(\mu_{I_{1}}, \ldots, \mu_{I_{\ell}}\right) \in \mathbb{Z}^{\ell}$ such that

$$
1 \leq \mu_{I_{i}} \leq\left(c_{I_{i}}-1\right) \operatorname{dim} X-1,
$$

there is a copy of the Lawson homology group of $X^{c(\mathcal{S})}$ (with shifted bidegree) in the decomposition of the Lawson homology group of $X[n]$. More precisely, we have the following summand in the decomposition of $L_{p} H_{k}(X[n])$ :

$$
L_{p-\|\underline{\mu}\|} H_{k-2\|\underline{\mu}\|}\left(X^{c(\mathcal{S})}\right)
$$

where $\| \underline{\mu \|}:=\sum_{i=1}^{\ell} \mu_{I_{i}}$ (notice that $\bigcap_{j=1}^{\ell} \Delta_{I_{j}} \cong X^{c(\mathcal{S})}$ ). Let $\mathcal{S}$ run through all the nests of $[n]$, we obtain all the direct summands of the decomposition of $L_{p} H_{k}(X[n])$, and the theorem follows.

Corollary 2.6 When $p=0$, Theorem 1.2 is reduced to a formula of the singular homology groups with integer coefficients for $X[n]$. 
Now we proceed to prove Theorem 1.3. A similar result for the Chow group and Chow motive of $X[n]$ was proved in [15]. For the readers' convenience we briefly recall the proof with the necessary adaptations to the present context. We need to recall the compositional formula of exponential generating functions. Let $f$ be a map from $\mathbb{Z}_{\geq 0}$ to a field $K$ of characteristic 0 . We denote by $E_{f}(t)$ the exponential generating function of $f$, ie,

$$
E_{f}(t)=\sum_{n \geq 0} f(n) \frac{t^{n}}{n !}
$$

Theorem 2.7 (Compositional formula [20, Theorem 5.1.4]) Let $K$ be a field of characteristic 0 . For any finite set $S$, denote by $\Pi(S)$ the set of ordered partitions of $S$. Given functions $f: \mathbb{Z}_{\geq 0} \rightarrow K$ with $f(0)=0$ and $g: \mathbb{Z}_{\geq 0} \rightarrow K$ with $g(0)=1$, define a new function $h: \mathbb{Z}_{\geq 0} \rightarrow K$ by $h(0)=1$ and

$$
h(|S|)=\sum_{\left\{B_{1}, \ldots, B_{k}\right\} \in \Pi(S)} f\left(\left|B_{1}\right|\right) f\left(\left|B_{2}\right|\right) \cdots f\left(\left|B_{k}\right|\right) g(k) \text {, for }|S|>0 .
$$

Then

$$
E_{h}(t)=E_{g}\left(E_{f}(t)\right)
$$

Proof of Theorem 1.3 By Theorem 1.2, it suffices to show that for any positive integers $m$ and $i$,

$$
\left.\sum_{c(\mathcal{S})=m} \sum_{\substack{\mu \in M_{\mathcal{S}} \\\|\underline{\mu}\|=i}} 1=\left[\frac{x^{i} t^{n}}{n !}\right] \frac{N^{m}}{m !}, \quad \text { (where } \mathcal{S} \text { is a nest of }[n]\right)
$$

which is equivalent to showing that for any positive integer $m$,

$$
\sum_{c(\mathcal{S})=m} \sum_{\underline{\mu} \in M_{\mathcal{S}}} x^{\| \mu} \|=\left[\frac{t^{n}}{n !}\right] \frac{N^{m}}{m !}, \quad(\text { where } \mathcal{S} \text { is a nest of }[n]) .
$$

First we consider the special situation $m=1$. Let $K$ be the polynomial ring $\mathbb{C}[x]$. Define $f: \mathbb{Z}_{\geq 0} \rightarrow K$ by

$$
f(n):=\sum_{c(\mathcal{S})=1} \sum_{\underline{\mu} \in M_{\mathcal{S}}} x^{\|\underline{\mu}\|}, \quad(\text { where } n>0 \text { and } \mathcal{S} \text { is a nest of }[n])
$$

and $f(0)=0$. We need to show that

$$
f(n)=\left[\frac{t^{n}}{n !}\right] N, \quad \text { for } n>0 .
$$


Fix an ordered partition $\left\{B_{1}, \ldots, B_{k}\right\} \in \Pi([n])$. Consider those nests $\mathcal{S}$ such that $c(\mathcal{S})=1$ and $B_{1}, \ldots, B_{k}$ are the maximal elements in

$$
(\mathcal{S} \backslash[n]) \cup\{\{1\}, \ldots,\{n\}\},
$$

in other words, consider those trees such that $B_{1}, \ldots, B_{k}$ are the labels of the children of the root. Each child of the root is the root of a subtree where we can use induction. Then we get a recursive relation

$$
f(n)=\sum_{\left\{B_{1}, \ldots, B_{k}\right\} \in \Pi([n])} f\left(\left|B_{1}\right|\right) f\left(\left|B_{2}\right|\right) \cdots f\left(\left|B_{k}\right|\right) g(k), \quad \text { for } n>1,
$$

where $f(1)=1$ and $g(k)$ is the function

$$
g(k)= \begin{cases}\sum_{i=1}^{d k-d-1} x^{i}=\left(x^{d k-d}-x\right) /(x-1) & \text { if } k>1 \\ 0 & \text { if } k=1 \\ 1 & \text { if } k=0\end{cases}
$$

Then

$$
\begin{aligned}
E_{g}(t) & =1+\sum_{k=2}^{\infty}\left(\frac{x^{d k-d}-x}{x-1}\right) \frac{t^{k}}{k !} \\
& =1+\frac{1}{x^{d}(x-1)} \sum_{k=2}^{\infty} \frac{\left(x^{d} t\right)^{k}}{k !}-\frac{x}{x-1} \sum_{k=2}^{\infty} \frac{t^{k}}{k !} \\
& =1+\frac{1}{x^{d}(x-1)}\left(\exp \left(x^{d} t\right)-1-x^{d} t\right)-\frac{x}{x-1}\left(e^{t}-1-t\right) \\
& =1+t+\frac{\exp \left(x^{d} t\right)-1}{x^{d}(x-1)}-\frac{x\left(e^{t}-1\right)}{x-1} .
\end{aligned}
$$

Define $h(n)$ to be the right hand side of 2.2 for $n>0$ and define $h(0)=1$. Then $h(n)$ and $f(n)$ only differ at $n=0,1$. Therefore

$$
E_{h}(t)=E_{f}(t)-t+1 .
$$

By the compositional formula of exponential generating functions (Theorem 2.7),

$$
E_{f}(t)-t+1=E_{g}\left(E_{f}(t)\right) .
$$

Denote $N:=E_{f}(t)$, we need to show that $N$ satisfies the identity in Theorem 1.3. This is indeed the case, since

$$
N-t+1=1+N+\frac{\exp \left(x^{d} N\right)-1}{x^{d}(x-1)}-\frac{x\left(e^{N}-1\right)}{x-1},
$$


or equivalently,

$$
(1-x) x^{d} t+\left(1-x^{d+1}\right)=\exp \left(x^{d} N\right)-x^{d+1} \exp (N) .
$$

Thus we have proved (3) in the special case $m=1$.

Now we prove (3) for $m>1$, ie, the case when forests have $m$ disjoint trees. First, notice that

$$
\sum_{c(\mathcal{S})=m} \sum_{\underline{\mu} \in M_{\mathcal{S}}} x^{\|\underline{\mu}\|}=\left[y^{m}\right] F(n)
$$

where $\quad F(n)=\sum_{\left\{B_{1}, \ldots, B_{k}\right\} \in \Pi([n])} f\left(\left|B_{1}\right|\right) f\left(\left|B_{2}\right|\right) \cdots f\left(\left|B_{k}\right|\right) y^{k}, \quad$ for $n \geq 1$

and $f$ is defined in (4). Indeed, fixing a partition $\left\{B_{1}, \ldots, B_{m}\right\} \in \Pi([n])$, we define $\mathfrak{S}$ to be the set of nests $\mathcal{S}$ such that $c(\mathcal{S})=m$ and the maximal elements in $\mathcal{S} \cup\{\{1\}, \ldots,\{n\}\}$ are $B_{1}, \ldots, B_{m}$. Define a function $n$ : (nests) $\rightarrow \mathbb{Z}$ by

$$
n(\mathcal{S}):=\text { number of leaves in the forest corresponding to } \mathcal{S} .
$$

For each connected component (which is a tree) in the forest corresponding to $\mathcal{S}$ we can use identity (3) in the case $m=1$. Then we have

$$
\sum_{\mathcal{S} \in \mathfrak{S}} \sum_{\underline{\mu} \in M_{\mathcal{S}}} x^{\| \mu} \underline{\|}=\prod_{i=1}^{m}\left(\sum_{\substack{c\left(\mathcal{S}_{i}\right)=1 \\ n\left(\mathcal{S}_{i}\right)=\left|B_{i}\right|}} \sum_{\underline{\mu} \in M_{\mathcal{S}_{i}}} x^{\| \mu} \underline{\mu}\right)=f\left(\left|B_{1}\right|\right) f\left(\left|B_{2}\right|\right) \cdots f\left(\left|B_{m}\right|\right) .
$$

Equation (5) immediately follows by adding up different partitions in $\Pi([n])$.

Applying again the compositional formula for $g(k)=y^{k}$, we obtain

$$
F(n)=\left[\frac{t^{n}}{n !}\right] \exp (y N)
$$

Therefore

$$
\left[y^{m}\right] F(n)=\left[y^{m}\right]\left[\frac{t^{n}}{n !}\right] \exp (y N)=\left[\frac{t^{n}}{n !}\right]\left[y^{m}\right] \exp (y N)=\left[\frac{t^{n}}{n !}\right] \frac{N^{m}}{m !} .
$$

This proves (3), and the theorem follows. 


\section{Deligne-Beilinson cohomology of Fulton-MacPherson space}

\subsection{Deligne-Beilinson cohomology}

Let $X$ be a complex manifold of complex dimension $d$. Let $\Omega_{X}^{k}$ the sheaf of holomorphic $k$-form on $X$. The Deligne complex of level $p$ is the complex of sheaves

$$
\underline{\mathbb{Z}}_{\mathcal{D}}(p): 0 \rightarrow \mathbb{Z} \stackrel{(2 i \pi)^{p}}{\rightarrow} \Omega_{X}^{0} \rightarrow \Omega_{X}^{1} \rightarrow \Omega_{X}^{2} \rightarrow \cdots \rightarrow \Omega_{X}^{p-1} \rightarrow 0
$$

The hypercohomology of the complex is called the Deligne-Beilinson cohomology of $X$ of level $p$ :

$$
H_{\mathcal{D}}^{*}(X, \mathbb{Z}(p)):=\mathbb{H}^{*}\left(X, \underline{\mathbb{Z}}_{\mathcal{D}}(p)\right)
$$

There is a multiplication of complexes

$$
v: \mathbb{Z}(p)_{\mathcal{D}} \otimes \mathbb{Z}(q)_{\mathcal{D}} \rightarrow \mathbb{Z}(p+q)_{\mathcal{D}}
$$

defined as

$$
v(x \bullet y)= \begin{cases}x \cdot y, & \text { if } \operatorname{deg} x=0, \\ x \wedge d y, & \text { if } \operatorname{deg} x>0 \text { and } \operatorname{deg} y=q>0, \\ 0, & \text { otherwise. }\end{cases}
$$

This gives a product structure on the Deligne-Beilinson cohomology as follows:

$$
\cup: H_{\mathcal{D}}^{k}(X, \mathbb{Z}(p)) \otimes_{\mathbb{Z}} H_{\mathcal{D}}^{k^{\prime}}(X, \mathbb{Z}(q)) \rightarrow H_{\mathcal{D}}^{k+k^{\prime}}(X, \mathbb{Z}(p+q)) .
$$

For details, the reader is referred to Esnault and Viehweg [5].

Let $X$ be an $d$-dimensional compact Kähler manifold. The Hodge filtration

$$
\cdots \subseteq F^{p} H^{k}(X, \mathbb{C}) \subseteq F^{p-1} H^{k}(X, \mathbb{C}) \subseteq \cdots \subseteq F^{0} H^{k}(X, \mathbb{C})=H^{k}(X, \mathbb{C})
$$

is defined by

$$
F^{p} H^{k}(X, \mathbb{C})=\bigoplus_{i \geq p} H^{i, k-i}(X) .
$$

We denote by $p_{X}^{k}$ the natural quotient map

$$
p_{X}^{k}: H^{k}(X, \mathbb{C}) \rightarrow H^{k}(X, \mathbb{C}) / F^{p} H^{k}(X, \mathbb{C}) .
$$

It was proved (see Esnault and Viehweg [5, Corollary 2.4] and Voisin [22, Proposition 12.26]) that we have the following long exact sequence:

$$
\begin{aligned}
\cdots \rightarrow H^{k-1}(X, \mathbb{C}) / F^{p} H^{k-1}(X, \mathbb{C}) & \rightarrow H_{\mathcal{D}}^{k}(X, \mathbb{Z}(p)) \\
& \rightarrow H^{k}(X, \mathbb{Z}) \rightarrow H^{k}(X, \mathbb{C}) / F^{p} H^{k}(X, \mathbb{C}) \rightarrow \cdots
\end{aligned}
$$


Now assume that $X$ is projective, and $Y, D$ are the same as defined in Section 2.1. Denote by $h$ the class of $\mathcal{O}_{\mathbb{P}\left(N_{Y / X}\right)}(-1)$ under the first Chern class $c_{1}: H^{1}\left(D, \mathcal{O}_{D}^{*}\right) \rightarrow$ $H_{\mathcal{D}}^{2}(D, \mathbb{Z}(1))$, ie, $h=c_{1}\left(\mathcal{O}_{\mathbb{P}\left(N_{Y / X}\right)}(-1)\right) \in H_{\mathcal{D}}^{2}(D, \mathbb{Z}(1))$ (see [5, page 88]).

The following proposition was proved in [5, Proposition 8.5].

Proposition 3.1 [5] The Deligne-Beilinson cohomology $H_{\mathcal{D}}^{k}(D, \mathbb{Z}(p))$ of the projective bundle $\pi: D \rightarrow Y$ is given by the isomorphism

$$
\bigoplus_{0 \leq j \leq r-1} H_{\mathcal{D}}^{k-2 j}(Y, \mathbb{Z}(p-j)) \cdot h^{j} \stackrel{\cong}{\longrightarrow} H_{\mathcal{D}}^{k}(D, \mathbb{Z}(p)),
$$

where $H_{\mathcal{D}}^{k-2 j}(Y, \mathbb{Z}(p-j)) \cdot h^{j}=\left\{\alpha \cup h^{j} \mid \alpha \in H_{\mathcal{D}}^{k-2 j}(Y, \mathbb{Z}(p-j))\right\}$.

Moreover, Barbieri Viale proved the following blow-up formula for Deligne-Beilinson cohomology:

Theorem 3.2 [2, Section A.3] Let $X, Y, D, \tilde{X}_{Y}, Y$ be as above. Then for each $p$, $k$ with $p \geq r \geq 0$, we have the isomorphism

(7) $\quad I_{p, k}:\left\{\bigoplus_{1 \leq j \leq r-1} H_{\mathcal{D}}^{k-2 j}(Y, \mathbb{Z}(p-j))\right\} \oplus H_{\mathcal{D}}^{k}(X, \mathbb{Z}(p)) \stackrel{\cong}{\longrightarrow} H_{\mathcal{D}}^{k}\left(\tilde{X}_{Y}, \mathbb{Z}(p)\right)$.

Remark 3.3 Barbieri Viale proved a general result, including the blow-up formula for étale cohomology, to Theorem 3.2.

Similarly, we compute the Deligne-Beilinson cohomology for Fulton-MacPherson configuration spaces.

Proof of Theorem 1.4 The proof is similar to the proof of Theorem 1.2: We use the fact that the Fulton-MacPherson configuration space $X[n]$ can be constructed by a sequence of blow-ups, and then successively apply the blow-up formula for Deligne-Beilinson cohomology (Theorem 3.2). We only point out the difference here: For each lattice point $\left(\mu_{I_{1}}, \ldots, \mu_{I_{\ell}}\right) \in \mathbb{N}^{\ell}$ such that $1 \leq \mu_{I_{i}} \leq\left(c_{I_{i}}-1\right) \operatorname{dim} X-1$, there is a copy of the Deligne-Beilinson cohomology of $X^{c(\mathcal{S})}$ in the decomposition of the Deligne-Beilinson cohomology of $X[n]$. More precisely, the summand for $\mu \in M_{\mathcal{S}}$ in the decomposition of $H_{\mathcal{D}}^{k}(X[n], \mathbb{Z}(p))$ is

$$
H_{\mathcal{D}}^{k-2\|\underline{\mu}\|}\left(X^{c(\mathcal{S})}, \mathbb{Z}(p-\|\underline{\mu}\|)\right) .
$$

Let $\mathcal{S}$ run through all the nests of $[n]$, we obtain all the direct summands of the decomposition of $H_{\mathcal{D}}^{k}(X[n], \mathbb{Z}(p))$, and then the theorem follows. 
Remark 3.4 By using the same method, we can compute the étale cohomology for Fulton-MacPherson configuration spaces.

Remark 3.5 The decomposition of Lawson homology (Theorem 1.2) and DeligneBeilinson cohomology (Theorem 1.4) of the Fulton-MacPherson configuration spaces can be generalized with no difficulty to the wonderful compactifications of arrangements of subvarieties, since the latter compactifications can also be constructed by a sequence of blow-ups along smooth centers (for the definition and the blow-up construction of these compactifications, see the second author's paper [16]).

\section{Comparing to homology}

(1) Let $X$ be a smooth $d$-dimensional complex projective variety. Then for each integer $k \geq 0$, there is an isomorphism of singular homology groups

$$
H_{k}(X[n]) \cong \bigoplus_{1 \leq m \leq n} H_{k}\left(X^{m}\right)^{\oplus\left[t^{n} / n !\right] N^{m} / m !}
$$

where $N:=N(x, t)$ is defined as in Theorem 1.3. This is a direct result of Theorem 1.3 and the Dold-Thom Theorem [4] which implies that $L_{0} H_{k}(V) \cong H_{k}(V)$ for any complex projective variety $V$. In particular, the integral singular homology of $X[n]$ depends only the integral singular homology of $X$. If there is no torsion in $H_{k}(X)$ for all $k \geq 0$, then there is no torsion in $H_{j}(X[n])$ for all $j \geq 0$. In particular, $H_{k}(C[n])$ has no torsion for any smooth complex projective curve $C$ since by the Künneth formula $H_{k}\left(C^{m}\right)$ has no torsion for all integers $k$ and $m$.

(2) If $X$ is a smooth complex cellular variety, ie, $X$ admits a filtration by closed subvarieties $\varnothing=X_{-1} \subset X_{0} \subset \cdots \subset X_{n}=X$ such that $X_{i}-X_{i-1} \cong \mathbb{C}^{m_{i}}$ for some positive integer $m_{i}$, then

$$
L_{p} H_{k}(X[n]) \cong H_{k}(X[n])
$$

for all integers $k \geq 2 p \geq 0$. This follows from Theorem 1.3 and a result of LimaFilho [17], since the product of cellular varieties is cellular.

(3) In contrast to the singular homology where $H_{k}(X[n])$ is always finitely generated for any $k, n$ and smooth projective variety $X$, the Lawson homology group $L_{p} H_{k}(X[n])$ can be infinitely generated even if $X$ is a smooth projective curve. This statement follows from Theorem 1.3 and a result of the authors [11] where we have found smooth algebraic curves $C$ such that $L_{p} H_{2 p}\left(C^{n}\right)$ is not finitely generated for $1 \leq p \leq n-2$ if $n \geq 3$. 


\section{Examples}

Example 5.1 (The Lawson homology group of $X[2]$ ) The morphism $\pi: X[2] \rightarrow X^{2}$ is a blow-up along the diagonal $\Delta_{12}$. Theorem 1.3 asserts

$$
L_{p} H_{k}(X[2]) \cong L_{p} H_{k}\left(X^{2}\right) \oplus\left\{\bigoplus_{j=1}^{d-1} L_{p-j} H_{k-2 j}(X)\right\} .
$$

Example 5.2 (The Lawson homology group of $X[3]$ ) Note that $X[3]$ is the blow-up of $X^{3}$ first along small diagonal $\Delta_{123}$, then along three disjoint strict transforms of diagonals $\Delta_{12}, \Delta_{13}$ and $\Delta_{23}$. Apply again Theorem 1.3 , we have

$$
\begin{aligned}
L_{p} H_{k}(X[3]) \cong L_{p} H_{k}\left(X^{3}\right) & \oplus\left\{\bigoplus_{j=1}^{d-1}\left(L_{p-j} H_{k-2 j}\left(X^{2}\right)\right)^{\oplus 3}\right\} \\
& \oplus\left\{\bigoplus_{j=1}^{2 d-1}\left(L_{p-j} H_{k-2 j}(X)\right)^{\oplus \min \{3 j-2,6 d-3 j-2\}}\right\} .
\end{aligned}
$$

Acknowledgements We would like to thank professor H Blaine Lawson and Mark de Cataldo for suggestions, conversations and all their help. We thank the referee for the suggestions to improve the presentation.

\section{References}

[1] S Axelrod, I M Singer, Chern-Simons perturbation theory. II, J. Differential Geom. 39 (1994) 173-213 MR1258919

[2] L Barbieri Viale, $H$-cohomologies versus algebraic cycles, Math. Nachr. 184 (1997) 5-57 MR1439168

[3] C De Concini, C Procesi, Wonderful models of subspace arrangements, Selecta Math. (N.S.) 1 (1995) 459-494 MR1366622

[4] A Dold, R Thom, Quasifaserungen und unendliche symmetrische Produkte, Ann. of Math. (2) 67 (1958) 239-281 MR0097062

[5] H Esnault, E Viehweg, Deligne-Beĭlinson cohomology, from: "Bělinson's conjectures on special values of $L$-functions", (M Rapoport, N Schappacher, P Schneider, editors), Perspect. Math. 4, Academic Press, Boston (1988) 43-91 MR944991

[6] E M Friedlander, Algebraic cycles, Chow varieties, and Lawson homology, Compositio Math. 77 (1991) 55-93 MR1091892 
[7] E M Friedlander, O Gabber, Cycle spaces and intersection theory, from: "Topological methods in modern mathematics (Stony Brook, NY, 1991)", (L R Goldberg, A V Phillips, editors), Publish or Perish, Houston, TX (1993) 325-370 MR1215970

[8] W Fulton, Intersection theory, second edition, Ergebnisse series (3) 2, Springer, Berlin (1998) MR1644323

[9] W Fulton, R MacPherson, A compactification of configuration spaces, Ann. of Math. (2) 139 (1994) 183-225 MR1259368

[10] W Hu, Birational invariants defined by Lawson homology arXiv:math. AG/0511722

[11] W Hu, L Li, Lawson homology, morphic cohomology and Chow motives arXiv: math.AG/0711.0383

[12] S Keel, Intersection theory of moduli space of stable $n$-pointed curves of genus zero, Trans. Amer. Math. Soc. 330 (1992) 545-574 MR1034665

[13] H B Lawson, Jr, Algebraic cycles and homotopy theory, Ann. of Math. (2) 129 (1989) 253-291 MR986794

[14] H B Lawson, Jr, Spaces of algebraic cycles, from: "Surveys in differential geometry, Vol. II (Cambridge, MA, 1993)", (C-C Hsiung, S-T Yau, editors), Int. Press, Cambridge, MA (1995) 137-213 MR1375256

[15] L Li, Chow Motive of Fulton-MacPherson configuration spaces and wonderful compactifications, to appear in Michigan Math. J. arXiv:math.AG/0611459

[16] L Li, Wonderful compactifications of arrangements of subvarieties, to appear in Michigan Math. J. arXiv:math.AG/0611412

[17] P Lima-Filho, Lawson homology for quasiprojective varieties, Compositio Math. 84 (1992) 1-23 MR1183559

[18] R MacPherson, C Procesi, Making conical compactifications wonderful, Selecta Math. (N.S.) 4 (1998) 125-139 MR1623714

[19] C Peters, Lawson homology for varieties with small Chow groups and the induced filtration on the Griffiths groups, Math. Z. 234 (2000) 209-223 MR1765879

[20] R P Stanley, Enumerative combinatorics. Vol. 2, Cambridge Studies in Adv. Math. 62, Cambridge Univ. Press (1999) MR1676282 With a foreword by G-C Rota and Appendix 1 by $\mathrm{S}$ Fomin

[21] DP Thurston, Integral expressions for the Vassiliev knot invariants arXiv: math. AG/9901110

[22] C Voisin, Hodge theory and complex algebraic geometry. I, Cambridge Studies in Adv. Math. 76, Cambridge Univ. Press (2002) MR1967689 Translated from the French original by L Schneps 
[23] C Voisin, Hodge theory and complex algebraic geometry. II, Cambridge Studies in Adv. Math. 77, Cambridge Univ. Press (2003) MR1997577 Translated from the French original by L Schneps

Department of Mathematics, MIT

Room 2-363B, 77 Massachusetts Avenue, Cambridge, MA 02139

Department of Mathematics, University of Illinois at Urbana-Champaign

Urbana, IL 61801

wenchuan@math.mit.edu, llpku@math.uiuc.edu

Received: 2 December 2008 Revised: 4 February 2009 